\title{
Sharing the IT Educational Experience of Developing 3D Applications for Medical Students Training
}

\author{
Stelian NICOLA ${ }^{\mathrm{a}, 1}$ and Lacramioara STOICU-TIVADAR ${ }^{\mathrm{a}}$ \\ ${ }^{a}$ Department of Automation and Applied Informatics \\ Politehnica University Timisoara, Romania
}

\begin{abstract}
The importance of using the new technologies to develop educational and training applications for medical students is given by the times we live in and also by the continued development of the IT industry. New concepts used in 3D applications such as gamification bring added value to the use of learning applications. The introduction of technologies-based on virtual and augmented reality contributes to increasing of the interactivity. This paper presents an overview of the students' experience in developing a complex 3D application for medical students. The application development methodology is explained by splitting the design and development of the 3D learning application into steps. The main users' benefits provided by such applications are increased interactivity and learning benefits, and the continuous availability of the application wherein virtual laboratories within a medical clinic are simulated.
\end{abstract}

Keywords. gamification, virtual reality, 3D application, medical education

\section{Introduction}

Currently, the IT industry growth is also due to the emergence of new technologies based on virtual reality (VR), augmented reality (AR), and mixed reality (MR). With the aid of such technologies, the IT industry has become involved in the vast majority of fields such as: education, medicine, healthcare, engineering. Many applications have been developed using such technologies which are meant to help the users learn or understand better certain systems and processes. Thus, new ideas have emerged, which are based on games forteaching and explaining certain procedures. Among the new concepts we focus on gamification. Gamification introduces the use of certain concepts which are used in applications or in games such as: granting bonuses (e.g. stars or experience points), timekeeping, displaying progress and certain suggestions meant to help the user to use the application [1]. Serious games, which, besides entertainment, are also aimed at educating or helping the users to understand certain concepts in various fields, are also based on gamification.

Numerous applications used in education or training may be found in the literature. Paper [2] presents an application supporting medical, bioinformatics, computer science and engineering students to train in a virtual environment, built for intensive care (ICU).

${ }^{1}$ Corresponding Author, Stelian NICOLA, Politehnica University Timisoara, P-ta Victoriei no. 2, Timisoara, Romania; E-mail: stelian.nicola@aut.upt.ro. 
Hardware-wise, the manner of operation of the devices as well as the processing of the resulting data are learned by the IT students. Medical students learn how to use the devices, but also how they should interact and react in certain situations with an ICU patient. The use of a 2D and 3D-based multimedia tool, of animations and video clips on certain systems of the human body (e.g. the circulatory system) is discussed in [3]. The benefit of such applications is given by the fact the students may learn without making errors or being afraid to make errors in real situations (e.g. dissecting a virtual human body). VR and AR-based applications are proposed for learning certain parts of the body or anatomical systems of the human body. Papers [4] and [5] discuss applications for learning the brain structure and, respectively, the skeletal system. AR is used to learn the heart structure and functions [6]. A 3D marker of the human body is proposed, that students may scan with the assistance of the application and then they may learn about the functions of the heart. VR is also used in the nursing field [7]. Based on VR applications, nurses may learn the steps they need to follow to change the bandage on a patient with a flesh wound. Visualizing a 3D virtual model, following each step to change the bandage as well as the provision of feedback and of instructions on how to change it introduces the concept of gamification in this application.

This paper will discuss the experience of the students enrolled in the Healthcare Information Systems (HIS) Master's degree of our university, in developing a complex 3D application based on VR. 20 students participated in the project. The application development methodology throughout a university semester (14 weeks) is also described. The motivation to develop such an application relies in the idea of providing learning and training applications to medical students who, in times like these (COVID 19) are prevented to physically attend laboratory classes.

\section{Methods and tools}

The application is divided into 5 modules and its purpose is to provide information on certain laboratories within a hospital or laboratory classes on human anatomical systems. The Unity editor together with several 3D models from the Unity asset store (e.g. the clinic model, the virtual nurse) was used for the application development, while the C\# language was used for the implementation of the functionalities. The application was developed for desktop, and it is based on VR technologies and gamification concepts.

The methodology employed to develop the HIS Master's projects is presented in the followings.

\subsection{Methodology}

Step 1: presentation of the project themes and the technologies employed within the project by the teaching staff. They presented several project themes and what they entail to the students. Among the themes that were approached there were: a radiology laboratory, an MRI laboratory, a blood collection laboratory, a laboratory for cell and tissue visualization and a laboratory for learning the locomotor (musculoskeletal) system.

Step 2: division of the students based on knowledge and preferences. Each student had to choose, based on their own knowledge and preferences, one of the following cases: C\# language programming knowledge, knowledge of use of the Unity 3D software package, minimal knowledge of the medical field (where minimal means medical knowledge of one of the themes that the teaching staff presented in step 1). 
Step 3: making groups of 4 students, where each member must have knowledge of one of the cases described in step 2. Thus, each group was built in such a way as to cover all the knowledge needed to realize the project theme.

Step 4: establishing the project theme for each team. Each team chose one of the 5 project themes presented in step 1: the radiology laboratory, the blood collection laboratory, the laboratory for the exploration and visualization of cells and tissue, the muscular system - learning laboratory and the skeletal system - learning laboratory.

Step 5: elaboration of a scenario by each team for the chosen project theme. The elaboration of the scenario consisted of the main functionalities that the application had perform. In addition, the stages that the application had to respect were also defined.

Step 6: identifying and researching the software functionalities of the application, but also a medical research on the methodology that must be respected when performing procedures within the laboratory.

Step 7: developing the application from a software and visual standpoint. Writing $\mathrm{C} \#$ scripts for the implementing the functionalities defined in steps 5 and 6 . Among the developed functionalities are: zooming in/out, moving, rotating 3D models, controlling the character from the keyboard, opening/closing laboratory doors, informing or helping the user via a help button, turning devices on and off. Finding, developing and using 3D models in the application scene. The students made searches and selected free 3D models of the characters (e.g. nurses, physicians, patients) and of devices or pieces of furniture used in a laboratory (e.g. microscope, X-ray machine, test tubes, computer, monitors). All 3D models were arranged in the laboratory based on its kind.

Step 8: testing the applications. This was performed by testing the software functionalities realized by each team. This was made by exchanging the applications. For instance, the team that developed the radiology laboratory tested the application of the team that developed the laboratory for the learning of the muscular system and vice versa. The testing was performed by the students using a test scenario. The main tests that were done: software function tests and generally user evaluation.

Step 9: software integration. All the laboratories developed by the team were integrated in a single 3D desktop application. Thus, a complex application was realized, wherein all the users may learn or explore various laboratories based on their needs. The entire application has a defined scenario that allows the users to control a character who may explore several laboratories within a hospital. The 3D application starts with the user visualizing a clinic, the clinic entry and the possibility to enter one of the laboratories developed by one of the teams and then explore them. A color was chosen for each team (red, blue, green, pink and yellow) for a better demarcation and visualization of the laboratories developed by each team. Thus, the door of each laboratory within the clinic is colored based on each team's color.

Figure 1 shows several images from the laboratories developed by the students. Therefore, figure 1 contains. (a) - radiology laboratory, figure (b) - muscular systemlearning laboratory, (c) - skeletal system-learning laboratory, (d) blood collection laboratory and (e) - cell and tissue-visualization laboratory. 


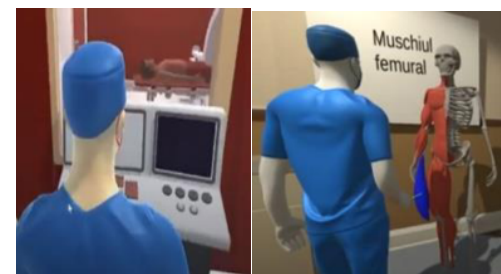

(a)

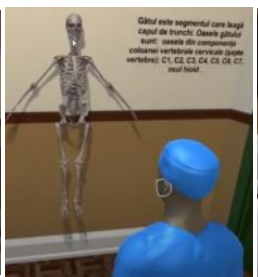

(c)

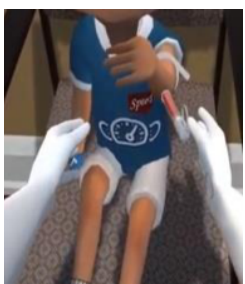

(d)

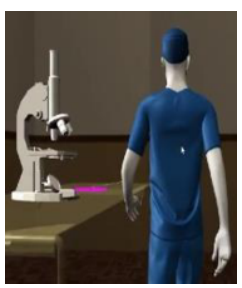

(e)

Figure 1. 3D Virtual Laboratories.

\section{Conclusions}

This paper highlighted the students' and staff experience in developing a complex 3D application meant to help medical students to learn certain laboratories more easily. Using a step or task-based methodology was efficient from the standpoint of delivering a functional application. The complexity of the application is given by the multitude of functionalities provided, by the 3D virtual environment, but also by the use of the virtual reality and gamification concepts.

In the future, we intend to test the application with medical students using new test methods by automatically identifying the user activity by means of a software service: Unity Analytics.

\section{Acknowledgment}

The authors would like to thank the Healthcare Information System Master's degree students from the Automation and Computers Faculty within the Politehnica University Timisoara, class of 2021, for their involvement and participation in the development of the complex 3D application.

\section{References}

[1] Lavoué E, Monterrat B, Desmarais M and George S, Adaptive Gamification for Learning Environments, IEEE Transactions on Learning Technologies. Jan.-March 2019;12(1):16-28.

[2] Forjan M, David V, Wagner M, Dolesch L, Lechner M and Sauermann S, Conceptualization of an ICU Infrastructure for Simulation Based Education in Medical Engineering \& eHealth, 2019 41st Annual International Conference of the IEEE Engineering in Medicine and Biology Society (EMBC).

[3] Majerník J, Szerdiová L,Preparation of Medical Students for Cadaveric Anatomy using Multimedia Education Tools, 2017 International Conference on Information and Digital Technologies (IDT).

[4] Wirza R, Afera CNS, Hod RB, Mobile Augmented Reality Application with Virtual Buttons as User Interaction and Stereoscopic View for Medical Learning, 2019 2nd International Conference on Applied Engineering (ICAE).

[5] Nicola S, Virag I, Stoicu-Tivadar L, VR Medical Gamification for Training and Education, 11th Annual Conference on Health Informatics Meets eHealth (eHealth), Austria, May 2017.

[6] Touel S, Mekkadem M, Kenoui M, Benbelkacem S, Collocated learning experience within collaborative augmented environment (Anatomy course), 2017 5th International Conference on Electrical Engineering - Boumerdes (ICEE-B).

[7] Choi KS, Virtual Reality Wound Care Training for Clinical Nursing Education: An Initial User Study, 2019 IEEE Conference on Virtual Reality and 3D User Interfaces (VR). 